\title{
Morphological Edge Detection on CT Images
}

\author{
A.Karthika ${ }^{1}$, Dr.D.Kiruab Jothi ${ }^{2}$ \\ ${ }^{1}$ Manonmaniam Sundaranar University,Tirunelveli, Tamilnadu, India,karthika.giri87@ gmail.com \\ 2Manonmaniam Sundaranar University,Tirunelveli, Tamilnadu, India, kirubajothi@ gmail.com
}

Received Date: January 01, 2022 Accepted Date : January 26, 2022 Published Date : February 07, 2022

\begin{abstract}
In this paper, a novel mathematical morphology edge detection algorithm is proposed to detect lungs CT medical image edge. It is a better method for edge information detecting and noise filtering than differential operation, which is sensitive to noise. And it is a better compromise method between noise smoothing and edge orientation, but the computation is more complex than general morphological edge detection algorithms.
\end{abstract}

Key words : CT Images, Morphology, Edge Detection.

\section{INTRODUCTION}

Sharp changes in image brightness are interesting for many reasons. Firstly, object boundaries often generate sharp changes in brightness - a light object may lie on a dark background, or vice versa. Secondly, reflectance changes often generate sharp changes in brightness which can be quite distinctive. Edges are the boundaries of objects in an image. The main characteristic of the edge pixels is the intensity change with that of its neighboring pixels. An edge is a property attached to an individual pixel and is calculated from the image function behavior in a neighborhood of that pixel. It is a vector variable with two components, magnitude and direction.

Edge detection has been an important subject in image processing because the edges correspond in general to important changes in physical or geometrical properties of objects in the scene. An edge fragment is a collection of edge points and an edge detector produces either a set of edge points or edge fragments. Edge detection is the identification of the intensity changes corresponding to the underlying physical changes. Edge detection is a sequence of processes (or single process) used to identify the edges in an image. Edge detection is the process that attempts to characterize the intensity (for gray image) in the image. Edge detection is by far the most common approach for detecting meaningful discontinuities in the gray level. Edge detecting an image significantly reduces the amount of data and filters out useless information, while preserving the important structural properties in an image. These reduced image data are used in subsequent image analysis operations for feature detection and object recognition. Also these edges (structure) of objects can be used for other image processing methods. So Edge Detection is an important and basic part of Digital Image Processing.

Most edge detection techniques apply a smoothing process before the extraction of intensity changes. It has been realized that the amount of smoothing can be adjusted by choosing a proper size for the smoothing operator. In many applications, a single scale of smoothing is not sufficient. In order to detect edges occurring naturally at a variety of spatial scales, multi-scale edge detection techniques are chosen. Multi-scale edge detection is accomplished by applying different sizes to an image, description of the signal changes can get at different scales. In general, the details of fine intensity changes can get from operators with small scale parameters and coarse information from operators with large scale parameters. The multi-scale analysis can be used not only to eliminate fine scale noise but also separate events at different scales arising from distinct physical processes. Many researchers have studied the multi-scale description of edges with the aim to see whether the original information can be recovered or not.

The two major problems associated with a multi-scale edge detection techniques are, the criteria to select the scales of operators for an image and how to effectively combine the edge information recovered at different scales. Selecting proper scale parameters for various gray images is a difficult problem. Exact edge detection (accurately delimiting physical changes) may not be possible due to the confounding effects of shadows, highlights, surface markings, texture and noise. Therefore, many researchers have concentrated on developing better algorithms for cleaning up edge maps produced by simplistic edge detectors.

\section{EDGE DETECTION METHODS}

2.1 First-Derivative Methods: Most edge detectors are based in some way on measuring the intensity gradient at a point in 
the image. Recall from vector calculus and differential geometry that the gradient operator is

$$
\nabla=\left[\begin{array}{l}
\frac{\partial}{\partial x} \\
\frac{\partial}{\partial y}
\end{array}\right]
$$

2.2Roberts Kernels: Since this chapter deals with differences between adjacent pixels, one way to find edges is to explicitly use a $\{+1,-1\}$ operator that calculates I (xi) - I (ax) for two pixels $\mathrm{I}$ and $\mathrm{j}$ in a neighborhood. Mathematically, these are called forward differences:

$$
\frac{\partial I}{\partial x} \approx I(x+1, y)-I(x, y)
$$

While these aren't specifically derivatives with respect to $x$ and $y$, they are derivatives with respect to the two diagonal directions. These can be thought of as components of the gradient in such a coordinate system. So, this kernel can calculate the gradient magnitude by calculating the length of the gradient vector

$\mathrm{g}=\sqrt{\left(g l^{*} f\right) 2+(g 2 * f) 2}$

\section{PREPARATION OF CT IMAGE}

Preparation for a CT scan is similar to X-rays and MRI scans. Most radiology clinics provide patients with a hospital gown, but otherwise all jewellery and items such as hats, belts, clips, and glasses must be removed as some objects have a detrimental effect on the image when scanned. In some cases, contrast agents are administered to image particular tissues more effectively. Many contrasts agents do contain iodine, which can provoke an allergic reaction in some patients. If you have an allergy to iodine or any other allergies, notify the nurse, technician or radiologists before the administration of the contrast agent. If you suspect you may be pregnant or you are pregnant, you must notify your doctor before the CT scan as this procedure does involve radiation and can be dangerous to a developing foetus. A CT scan is very similar to a MRI scan.

You will be asked to lie on the table in a still position and the table will move into the tunnel. During the scan, the table will move a small distance every few seconds to reposition you for the next scan. During the scanning, the machine may make buzzing or click sounds as it moves. You will be alone in the scanning room, but the radiologist conducting the scan will be able to see you through a window into the room and communicate with you via intercom. The scan can last from 30-90 minutes, during which you will be asked to lie very still in the scanner which for some can cause anxiety or claustrophobia. If you suffer from a fear of small spaces (claustrophobia), inform your doctor and the radiologists and a sedative can be administered in appropriate conditions.
After the scan, the details of the results may be shared by the radiologist or referred back to your doctor. Your day can continue as normal however it is recommended if you were given a contrast agent to drink water to flush your body of the agent.

\section{THE PROPOSED MORPHOLOGICAL EDGE DETECTION ALGORITHM}

Morphological edge detection algorithm selects appropriate structuring element of the processed image and makes use of the basic theory of morphology including erosion, dilation, opening and closing operation and the synthesization operations of them to get clear image edge. In the process, the synthesized modes of the operations and the feature of structuring element decide the result of the processed image. Detailed saying, the synthesized mode of the operations reflects the relation between the processed image and origin image, and the selection of structuring element decides the effect and precision and the result. Therefore, the keys of morphological operations can be generalized for the design of morphological filter structure and the selection of structuring element. In medical image edge detection, we must select appropriate structuring element by texture features of the image. And the size, shape and direction of structuring element must have been considered roundly. Usually, except for special demand, we select structuring element by $3 \times 3$ square.

By the operation features of morphology, erosion and dilation operations satisfy:

$F \Theta B \subseteq F \subseteq F \oplus B$

Opening and Closing Operations are:

$F \circ B \subseteq F \subseteq F \bullet B$

In the field of morphological edge detection, the following algorithms are used for image edge detection. The edge of image $\mathrm{F}$, which is denoted by $\operatorname{Ed}(\mathrm{F})$, is defined as the difference set of the dilation domain of $\mathrm{F}$ and the domain of $\mathrm{F}$. This is also known as dilation

$E_{d}(F)=(F \oplus B)-F$

Similarly, the closing top-hat transformation of image F, which is denoted by THc (F), can also be defined as the difference set of the closing domain of $\mathrm{F}$ and the domain of $\mathrm{F}$. It is defined as

$$
T H_{c}(F)=(F \bullet B)-F \text {. }
$$

The top-hat transformation, which owes its original name to the use of a cylindrical or parallelepiped structuring element function with a flat top, is useful for enhancing detail in the presence of shading.

The effect of erosion and dilation operations is better for image edge by performing the difference between processed image and original image, but they are worse for noise filtering. As opposed to erosion and dilation, opening and closing operations are better for filtering. But because they utilize the complementarity of erosion and dilation, the result of processed image is only correlative with the convexity and 
concavity of the image edge. Accordingly, what we get is only the convex and concave features of the image by performing the difference between processed image and original image, but not all the features of image edge.

In this paper, a novel mathematical morphology edge detection algorithm is proposed. Opening-closing operation is firstly used as preprocessing to filter noise. Then smooth the image by first closing and then dilation. The perfect image edge will be got by performing the difference between the processed image by above process and the image before dilation. The following is the novel algorithm:

$$
M \bullet B \oplus B-M \bullet B
$$

where

$$
M=(F \bullet B) \circ B
$$

\section{EXPERIMENTAL RESULTS AND ANALYSIS}

In this section, the proposed morphological edge detection algorithm is compared with a variety of existing methods for edge detection. In the Results, figure.1 is the original lungs CT image. Fig.2 is the original lungs CT image with salt and pepper noise. Fig. 3 and Fig.4 are the results of processed lungs CT images after respectively applying Laplacian of Gaussian operator and Sobel edge detector. Fig.5 and Fig.6 are the lungs CT images processed by morphological gradient operation and dilation residue edge detector which are denoted by the equations (9) and (7) respectively. Fig.7 is the lungs CT image processed by the novel morphological edge detector proposed in this project by equation (9).

According to the experiment results shown in Fig. 3 and Fig.4, Laplacian of Gaussian operator and Sobel edge detector detect the lungs edges successfully, but Sobel edge detector fail to detect the outer edge of body, and both of them can't filter the noise. By Fig.5 and Fig.6, the morphological gradient operation and dilation residue edge detector are succeeding in lungs and body edges detection, and the detected edges are clearer than the edges detected by Laplacian of Gaussian operator and Sobel edge detector. But both of them fail to filter the noise in despite of the latter is better for noise filtering than the former. By Fig.7, the novel morphological edge detector proposed in this project is succeed in lungs and body edges detection, but more important than template-based edge detection algorithm and general morphological edge detection algorithm mentioned before, it also filters the noise successfully. Newer algorithm is implemented and tested with the advanced research tool of IDL. It is nothing but, Interactive data Language. All Mathematical Equations are implemented in individual functions and CT Edges are extracted in function 'Main'. Future Addition of Function will be easy and produce the high intensity edges are well enhanced. The results are

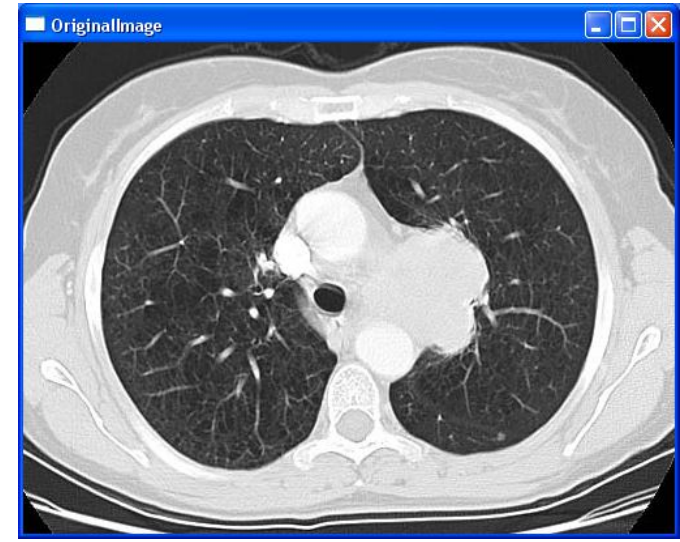

Figure 1: Original Image

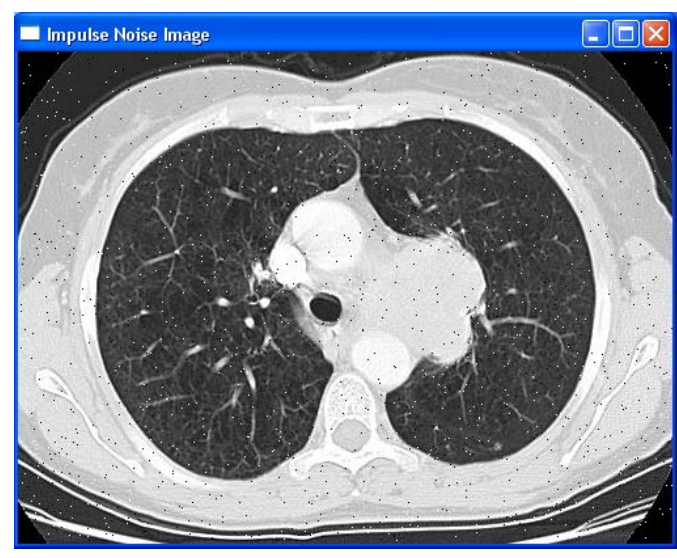

Figure 2: Salt and Pepper Noise CT Image

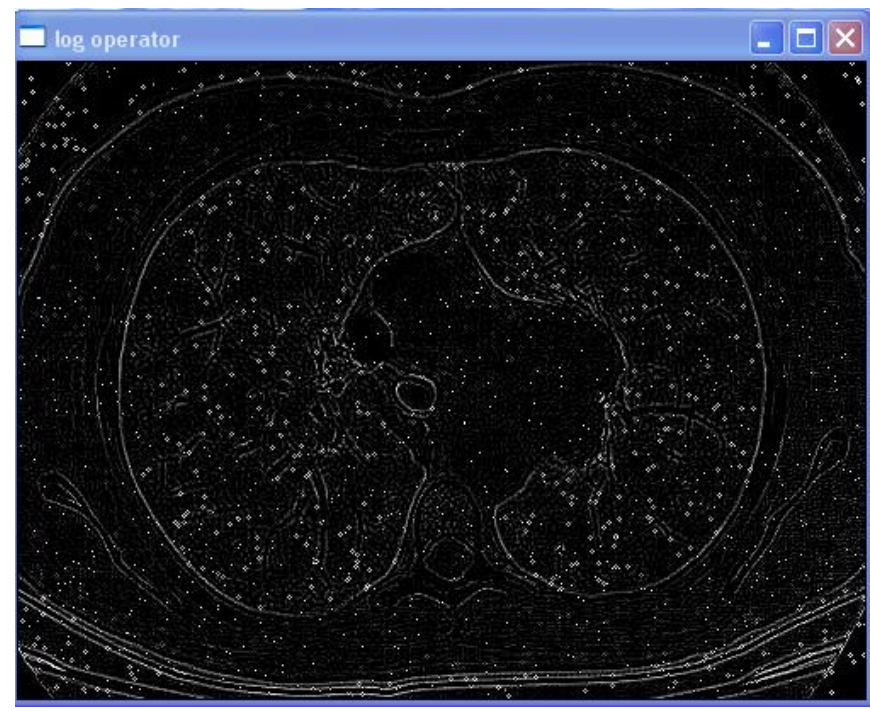

Figure.3: Lungs CT image processed by Laplacian of Gaussian operator 


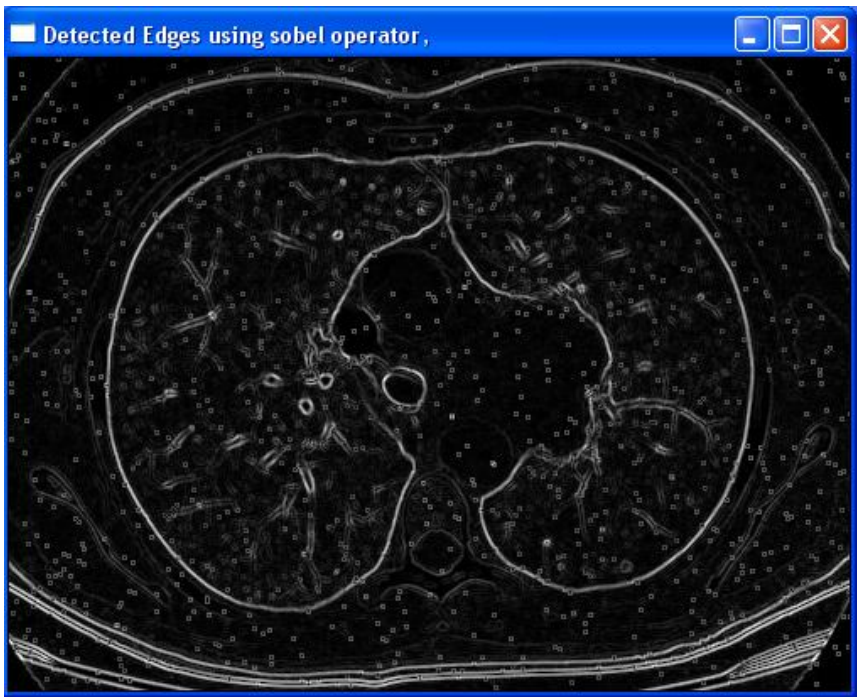

Figure. 4: Lungs CT image processed by Sobel detector.

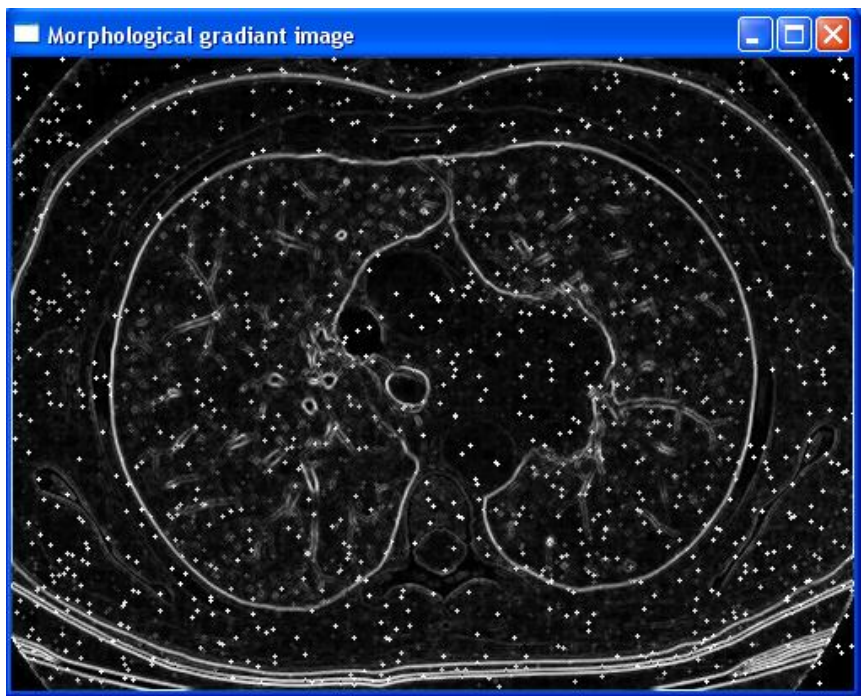

Figure 5: Lungs CT image processed by morphological gradient operation

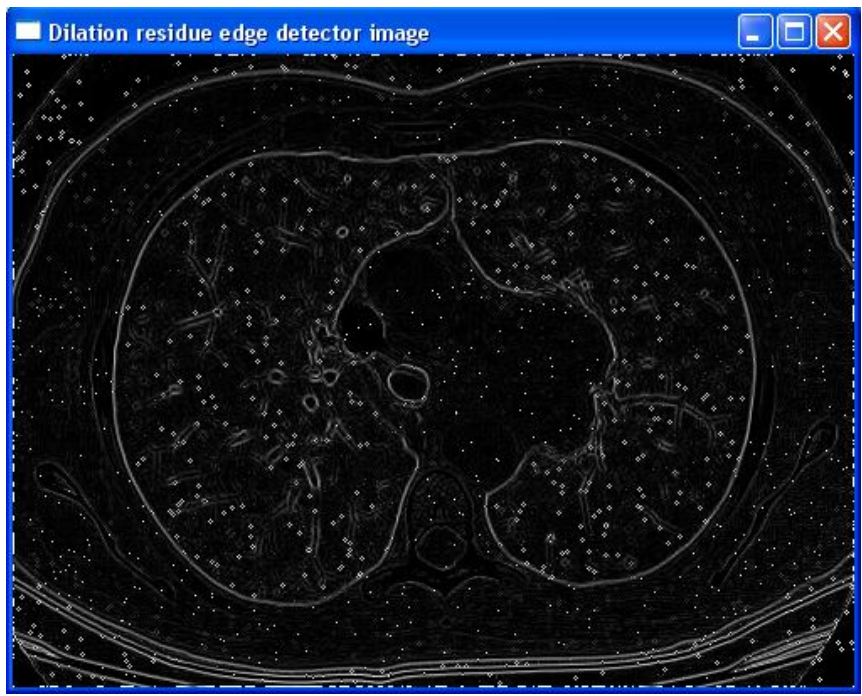

Figure 6: Lungs CT image processed by dilation residue edge detector.

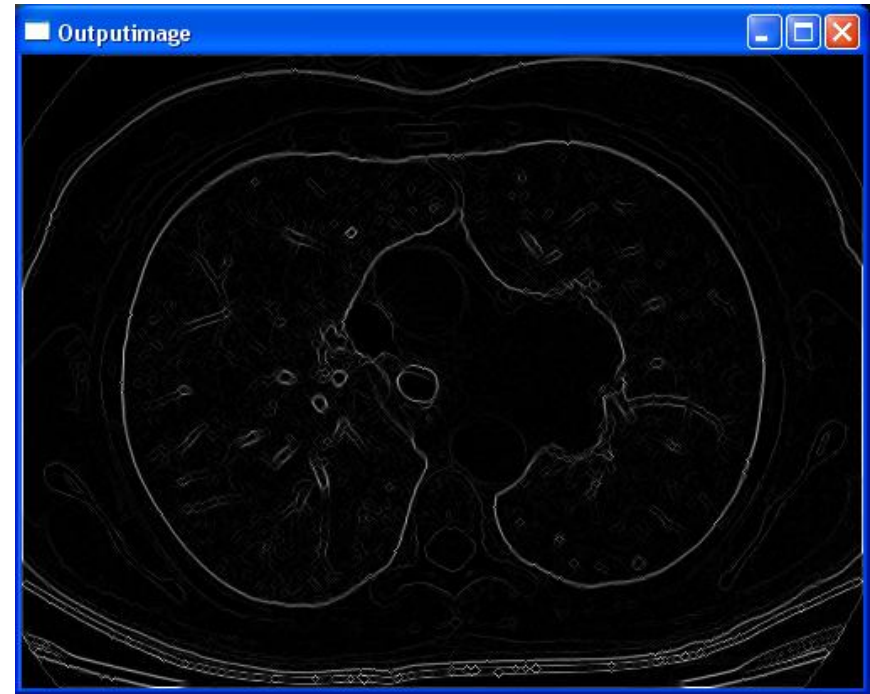

Figure .7 :Lungs CT image processed by the novel Morphological edge detector.

\section{CONCLUSION}

In this research, a novel mathematic morphological algorithm is proposed to detect lungs CT medical image edge. The experimental results show that the algorithm is more efficient for medical image denoising and edge detecting than the usually used template-based edge detection algorithms such as Laplacian of Gaussian operator and Sobel edge detector, and general morphological edge detection algorithm such as morphological gradient operation and dilation residue edge detector.

\section{REFERENCES}

1. M.I. Rajab, M.S. Woolfson, and S.P. Morgan, "Application of region- based segmentation and neural network edge detection to skinlesions,"

Computerized Medical Imaging and Graphics, vol. 28, pp. 61-68, 2004.

2. H. Tang, E.X. Wu, Q.Y. Ma, D. Gallagher, G.M. Perera, and T. Zhuang,"MRI brain image segmentation by multi-resolution edge detection and region selection," Computerized Medical Imaging and Graphics, vol 24, pp. 349-357, 2000.

3. Huertas, A. and Medioni, G., "Detection of intensity changes with sub pixel accuracy using Laplacian-Gaussian masks," IEEE Trans. On Pattern Analysis and Machine Intelligence, PAMI, vol. 8, pp. 651-664, 1986.

4. Maragos P., "Differential Morphology and Image Processing," IEEE Trans Image Processing, vol. 5, pp. 922-937, June 1996.

5. Ortiz F, and Torres F, "Vectorial Morphological Reconstruction for Brightness Elimination in Colour Images," Real-Time Imaging, vol. 10, pp. 379-387, June 2004.

6. Jing Xiao-jun, Yu Nong, and Shang Yong, "Image Filtering Based on Mathematical Morphology and Visual 
A.Karthika et al., International Journal of Emerging Trends in Engineering Research, 10(2), February 2022, 43 - 47

Perception Principle," Chinese Journal of Electronics, vol. 13, pp. 612-616, April 2004.

7. Richard A P, “A New Algorithm for Image Noise Reduction Using Mathematical morphology," IEEE Transaction on Image Processing, vol. 4, pp. 554-568, March (1995).

8. Chen T., and Wu Q.H., Rahmani-Torkaman R., Hughes J., "A pseudo top-hat mathematical morphological approach to edge detection in dark regions," Pattern Recognition, vol. 35, pp. 199-210, 2002.

9. Rivest Jean, "Morphological Operators on Complex Signals," Signal Processing, vol. 84, pp. 133-139, January 2004.

10. Lee J.S.J., Haralick R.M., and Shapiro L.G., "Morphological Edge Detection," IEEE J. Robot. Automat, vol. 3, pp. 142-156, February 1987. 